\title{
Spatial Diversity Benefits by Means of Induced Fading
}

\author{
Daniele Puccinelli and Martin Haenggi \\ Network Communication and Information Processing Laboratory \\ Department of Electrical Engineering \\ University of Notre Dame \\ \{dpuccine, mhaenggi\}@nd.edu
}

\begin{abstract}
Multipath fading heavily contributes to the unreliability of wireless links and is normally seen as a negative phenomenon hindering proper radio communication. However, fading can also improve the chances of reliable communication over channels that would otherwise be unusable, as our experimental evidence shows. In the context of wireless sensor networks, we show that limited motion of the base station may be used to obtain a considerable spatial diversity benefit through the exploitation of induced fading. We compare our limited mobility approach to multi-antenna systems, which are commonly used to achieve spatial diversity, and we illustrate these concepts with the help of experimental results. Moreover, we analyze how spatial diversity relates to network lifetime.
\end{abstract}

\section{INTRODUCTION}

In this paper, we characterize the concept of induced fading, i.e., the alteration of the multipath fading pattern in a given environment, which may lead to a gain in the received power. We focus on indoor deployments of wireless sensor networks, and assume the sensing nodes to be static. Fading is typically caused by activity in the surroundings of the nodes, as moving obstacles (people or objects) alter the signal propagation patterns causing extra reflections. Motion in the vicinity of nodes causes small-scale fading, resulting in variations of up to $\pm 5 \mathrm{~dB}$. Larger variations can be caused by the motion of objects through line-of-sight (LOS) paths, which generates shadowing effects. In situations where the signal normally does not have enough power to be detected by the receiver, fading can be advantageous, as it can cause a temporary increase in signal power. This typically happens when an obstacle creates a favorable geometry by producing one or more multipath components whose path length is shorter than the path length of previously existing components. An obstacle can also create a particularly favorable phase alignment of the multipath components leading to their constructive superposition.

Motion in the environment is sufficient to create fading, but fading may also be artificially induced by adding mobility to the network in the form of a mobile base station continuously moving within a small area. Temporal variations in the channel seen by such a mobile agent are therefore induced by its motion; in this sense we speak of induced fading, which has been studied in [1] in the context of multi-antenna systems. In this paper we show that spatial benefits may be obtained by means of the induction of fading through limited mobility, which we define as the ability of the base station to move around within an area of the order of $\lambda^{2}$; since signal strength measurements are essentially uncorrelated over spatial displacements of the receiver of about $\lambda / 2$, motion of such a limited scope is sufficient to obtain a significant spatial diversity benefit through induced fading. Spatial diversity is more commonly achieved by means of multi-antenna systems; a comparison between these two approaches is performed.

Network lifetime is particularly critical in wireless sensor networks due to their untethered nature, which calls for the minimization of ongoing maintenance. In the case of a many-to-one traffic paradigm where all nodes have the same probability of injecting data into the network, the nodes located one hop away from the base station (critical nodes) are prone to be overburdened by an excessive workload. In fact, all paths to the base station go through such nodes, which are forced to relay a large amount of traffic and also have to send their own data; the fewer the critical nodes, the higher their overload [2]. With limited mobility, induced fading causes the connectivity properties of a network to vary depending on the position of the base station. If we let the base station move to different positions, we intuitively expect it to be able to communicate with a different set of nodes 
from each new position, thus dynamically extending the number of one-hop neighbors and therefore the critical area. Hence, the extension of the critical area can redistribute the workload, allowing for an improved energy balancing and an extended network lifetime. The critical area can be extended through the spatial diversity benefit from limited mobility or multi-antenna systems; another contribution of this paper is the development of analytical tools to quantify the critical area extension.

The remainder of this paper is organized as follows. After an overview of related work in Section II, we present our geometric fading model in Section III. In Section IV, we show how limited mobility of the base station can induce fading and achieve a spatial diversity benefit, and we compare this approach to multi-antenna solutions. Finally, in Section V, we relate the spatial diversity gain from limited mobility and multi-antenna systems to network lifetime.

\section{RELATED WORK}

This paper fuses three main ingredients: fading, spatial diversity, and mobility in sensor networks. Fading is often seen as harmful, and spatial diversity techniques are exploited to contrast it. The body of literature on fading and multi-antenna approaches to the achievement of spatial diversity is fairly rich and diverse; a good overview can be found in [3]. As for the impact of fading in sensor networks, the Rayleigh fading approach to link modeling in [4] and [5] was particularly influential to our framework.

The idea of inducing fading to achieve spatial diversity benefits is not new to this paper. In particular, it is pointed out in [1] that whereas fading is traditionally seen as a source of unreliability to be mitigated, it can instead be considered as a source of randomization to be exploited. The authors focus on cellular systems and introduce opportunistic beamforming, promoting the use of multiple transmit antennae to induce large and fast fluctuations in environments with little scattering. An interesting element of novelty in our approach is the use of limited mobility to induce positive fading gains.

The introduction of controlled mobility in wireless sensor networks has been the focus of several research efforts. In [6] the possibility of using a mobile node acting as an information sink is indicated. In [7], mobile nodes are used as message ferries which permit message exchanges between disconnected nodes. Mobile agents are also at the center of the SENMA architecture [8], [9]: they communicate with simple sensor nodes over a single hop by minimizing the path loss and handle primary network functions. It is worth mentioning that data mules are already being used in existing sensor network deployments [10], [11]. However, our focus is on limited mobility for spatial diversity gain, whereas these approaches typically attempt the minimization of the large-scale path loss by having the mobile agent approach the individual nodes, which requires motion across the whole network area. The benefits of limited mobility are acknowledged in [12], where the added value of a mobile sink is recognized and analyzed.

\section{A GEOMETRIC FADING MODEL}

We start by introducing a deterministic multipath fading model which uses similar concepts as the tenray model [13] as well as ideas deriving from twodimensional ray tracing [14]. We consider a 2D geometry, assume isotropic antennae, neglect refractions and multiple reflections, and thus only consider paths with a single reflection. Aside from the direct LOS path, we consider four other paths produced by the reflections of the radio waves off the walls or obstacles, indicated in Figure 1 as $T$ (top), $B$ (bottom), $L$ (left), and $R$ (right) with obvious reference to the geometry of the layout. In a zero-interference network, the signal received at node $y$ upon transmission from node $j$ can be written as

$$
r(t)=\operatorname{Re}\left\{\frac{\lambda \sqrt{G} e^{j 2 \pi f_{c} t}}{4 \pi} \sum_{i=0}^{4} \frac{s_{i} u\left(t-\tau_{i}\right) e^{-j 2 \pi d_{i} / \lambda}}{d_{i}}\right\},
$$

where $P_{\mathrm{tx}}$ is the transmit power, $\lambda$ is the wavelength corresponding to the carrier frequency $f_{c}, G$ is the product of the gains of the transmit and receive antennae (which we assume to be omnidirectional), $u(t)$ is the baseband equivalent of the transmitted signal, $B_{u}$ is its bandwidth, and $d_{i}$ is the length of the $i$-th path ( $d_{0}=d_{L}$ is the length of the LOS component). Finally, $\tau_{i}=\lambda\left(d_{i}-d_{L}\right) / c$ is the time delay of the $i$-th ray with respect to the LOS component, and the attenuation coefficient $s_{i}$ represents the product of the refraction coefficients of the surfaces that the $i$-th ray penetrates and the reflection coefficients of the obstacles that it encounters. We assume flat fading $\left(\tau \ll B_{u}^{-1}\right)$, so that $u(t) \approx u\left(t-\tau_{i}\right)$ for every $i$, and the received power may be written as

$$
P_{\mathrm{rx}}=P_{\mathrm{tx}} G\left(\frac{\lambda}{4 \pi}\right)^{2}\left|\sum_{i=0}^{4} \frac{s_{i} e^{-j 2 \pi d_{i} / \lambda}}{d_{i}}\right|^{2}=P_{\mathrm{tx}} L_{p}(\mathbf{y}, \mathbf{j})
$$

where $L_{p}$ is the path loss function, which captures the power decay due to propagation through the wireless 


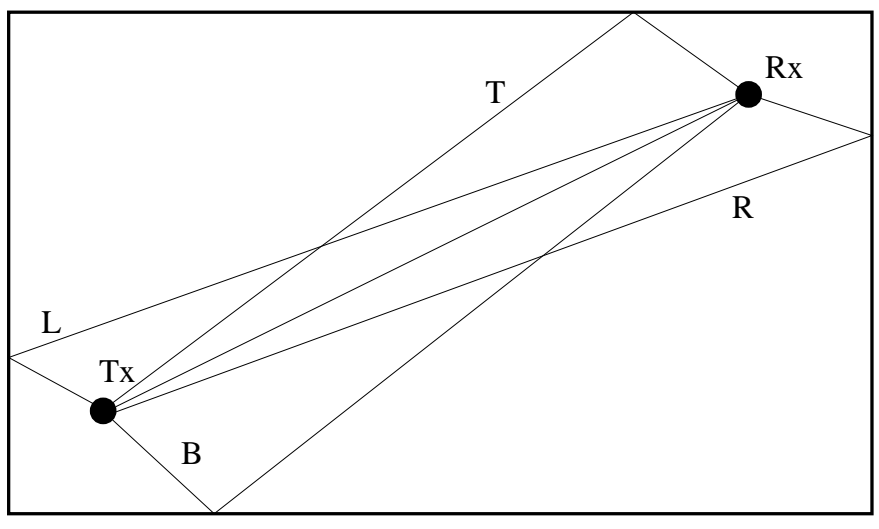

Fig. 1. An example of the geometry used in our model. We consider 4 paths in addition to the LOS path (indicated by $D$ ): $T$ (top), $B$ (bottom), $L$ (left), and $R$ (right).

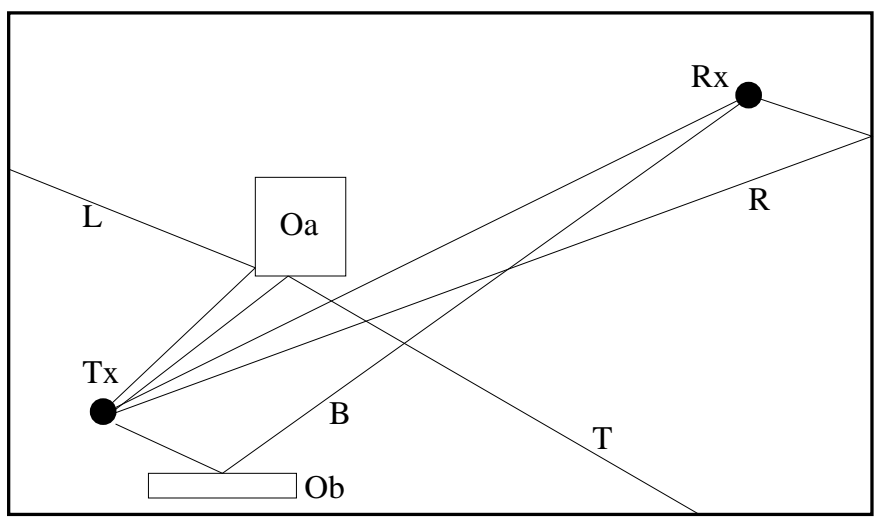

Fig. 2. The presence of obstacles $O a$ and $O b$ modifies the paths: multipath components $T$ and $L$ can no longer reach the receiver due to $O a$, and the path length of $B$ is shortened because of $O b$.

medium and depends on $\mathbf{y}$ and $\mathbf{j}$, the spatial coordinates of nodes $y$ and $j$.

Since the attenuation coefficients and the length of the paths depend on the geometry of the surroundings, the network layout, and the presence of obstacles in the environment, the received signal is often modeled as a random process parametrized by the internode distance $d_{L}$. Assuming a Rayleigh link model, the power received by node $y$ at a distance $d_{L}$ from node $j$ follows an exponential distribution given by

$$
f_{P_{\mathrm{rx}}}(x)=\frac{1}{\overline{P_{\mathrm{rx}}}\left(d_{L}\right)} \exp \left(-\frac{x}{\overline{P_{\mathrm{rx}}}\left(d_{L}\right)}\right),
$$

where $\overline{P_{\mathrm{rx}}}\left(d_{L}\right)=q P_{\mathrm{tx}} d_{L}^{-\alpha}$ denotes the average received power (the prefactor $q$ depends on the wavelength and the antenna gain); thus, the envelope of the received signal is Rayleigh-distributed. We say that a receiver located at a distance $d_{L}$ from a transmitter is in a good fading spot if it lies in a location where $P_{\mathrm{rx}} \gg \overline{P_{\mathrm{rx}}}\left(d_{L}\right)$. Fading may undoubtedly be harmful, as it may affect the RF signal with deep fades; nevertheless, positioning terminals in good fading spots results in a better channel than what would be expected from the large-scale path loss.

Obstacles in the environment may not only block off and attenuate paths, but also shorten them, depending on the geometry of the environment and the nature of the obstacles. A typical geometry with obstacles is shown in Figure 2. Using a threshold model (the physical model in [15]), a transmission from node $j$ to node $y$ is successful if the received signal strength at $y$ is above a threshold $\Theta$ that depends on the noise floor and the communication hardware. In the experiments presented in this paper we mainly use MICAz motes from CrossBow, equipped with an Atmel ATmega128L MCU and an IEEE 802.15.4compliant $2.4 \mathrm{GHz}$ Chipcon $\mathrm{CC} 2420$ radio which takes advantage of spread spectrum techniques to increase channel reliability and noise tolerance by spreading the signal over a wider range of frequencies; for this radio, our experience dictates that $\Theta$ is comprised between -85 and $-90 \mathrm{dBm}$. In all the experiments in this paper, a transmit power of $-10 \mathrm{dBm}$ is used (and the same transmit power is assumed in the numerical examples). In Section IV we use our geometric fading model as a handy tool to clarify the notion of induced fading, and to illustrate the spatial diversity benefits of limited mobility. Since multi-antenna systems are typically used to obtain spatial diversity benefits, we compare an example of such a system to limited mobility to highlight the analogies and the differences.

\section{INDUCING FADING WITH MOBILITY}

\section{A. Induced Fading}

Figure 3 shows a signal strength acquisition performed over an 802.11b wireless link between a PC and a CrossBow Stargate gateway. If the reception threshold $\Theta$ of the receiver is $-80 \mathrm{dBm}$, no reception is possible except when motion occurs in the surroundings of the nodes between time $20 \mathrm{~s}$ and $35 \mathrm{~s}$. Therefore, it is clear from this experimental example that fading may enable packet reception over an otherwise unusable channel.

Figure 4 shows an example of induced fading obtained with MICAz hardware. As illustrated in Figure 5, a transmitter in a room sends packets of fixed size every $15 \mathrm{~ms}$ to a receiver located in the adjacent hallway (about $12 \mathrm{~m}$ away); the LOS path suffers multiple interruptions. In Figure 4, we show whether a packet is received or not at each time instant. Due to the unfavorable positioning of the nodes and the geometry 


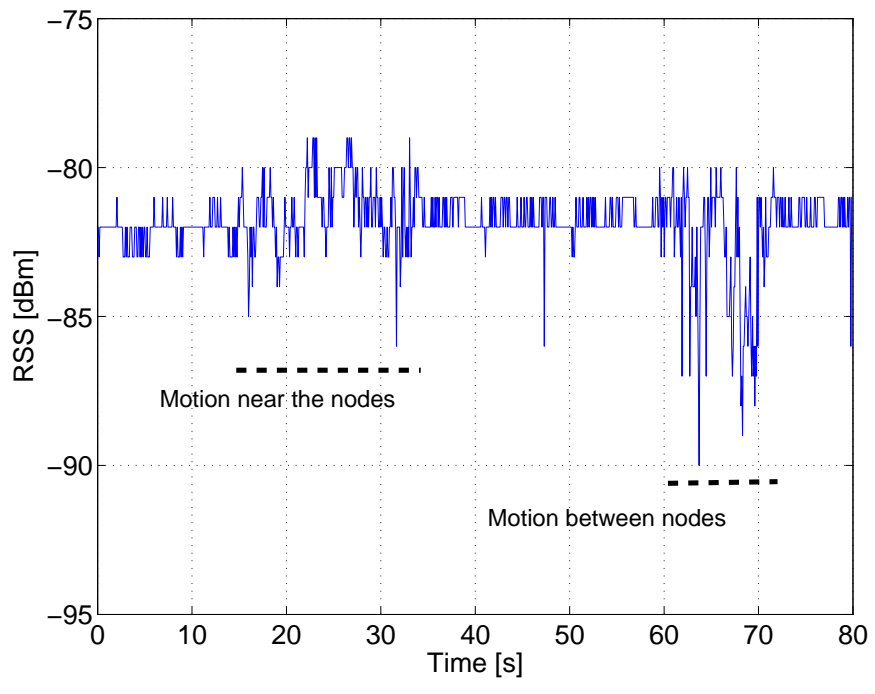

Fig. 3. Motion between the nodes produces shadowing effects, whereas motion near the nodes causes multipath fading. In this example, with a reception threshold $\Theta$ of $-80 \mathrm{dBm}$ induced fading allows some degree of reception between time $20 \mathrm{~s}$ and $35 \mathrm{~s}$. This acquisition (at 20 samples per second) was performed over an 802.11 b wireless link.

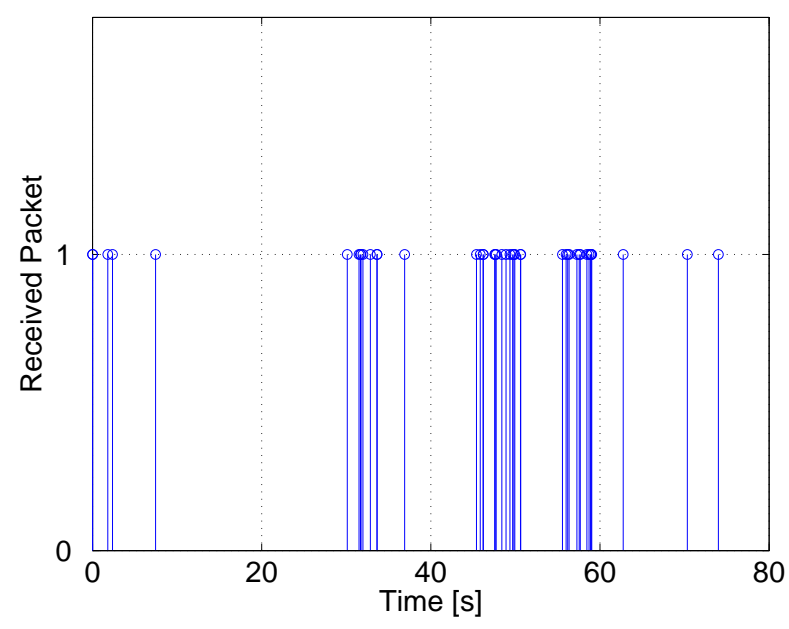

Fig. 4. Packet reception is enabled by layout changes due to the motion of individuals which induces a favorable fading pattern.

of their surroundings, nothing can be received under normal conditions. By passing through or stopping in particularly favorable spots, individuals create a special fading pattern which makes reception possible: induced fading enables communication. In addition to illustrating this with experimental evidence, we wish to provide some simple intuition. In the example in Figure 6, in the presence of obstacles $O a, O b$, and $O c$, our model predicts that no packet reception is possible (in this example for simplicity we assume no signal penetration through

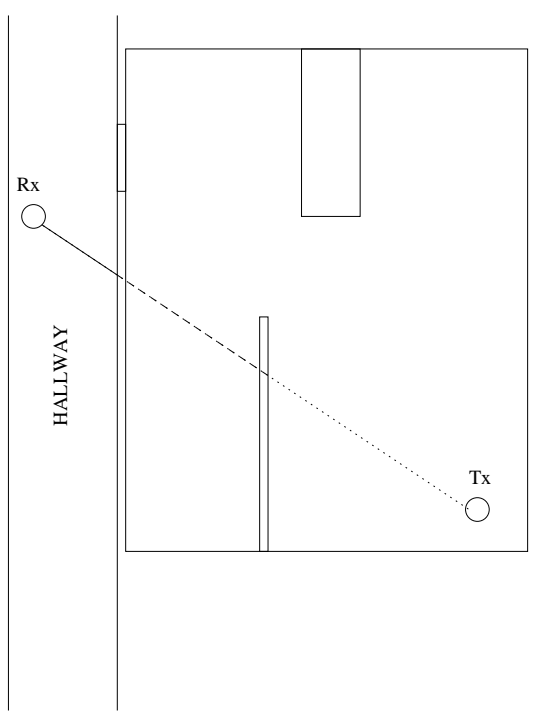

Fig. 5. Geometry of induced fading demonstration. The LOS is interrupted by a wall and a thin obstacle inside the room.

obstacles). In the presence of obstacle $O d$ (example in Figure 7), however, our simple geometric model predicts a received signal strength of $-74.6 \mathrm{dBm}$, more than $10 \mathrm{~dB}$ above the typical value for the reception threshold. Therefore, $O d$ induces a favorable fading pattern which offsets the negative impact of the other three obstacles.

\section{B. Limited Mobility and Multi-Antenna Systems}

Fading may be induced with the motion of one of the terminals; as we already anticipated, our focus is on limited mobility as a means of achieving a spatial diversity benefit. Limited mobility exploits spatial diversity in a dynamic and continuous fashion, contrarily to multi-antenna systems that sample the path loss function at spatial intervals which typically equal about $\lambda / 2$, the physical separation of neighboring antennae on an array. This form of spatial undersampling of the path loss function implies that the overall reception probability in multi-antenna systems depends strongly on the particular positioning of the antenna array. Both approaches require extra hardware, but adding extra antennae to a wireless terminal is in general cheaper than making the terminal mobile. In the case of a lowerend node, however, adding extra antennae is impractical. Antennae need to be spaced by at least $0.4 \lambda$ in order for them to take uncorrelated channel samples [16]; in the case of MICA2 Berkeley motes operating at a carrier frequency of $433 \mathrm{MHz}$, antennae would need to be at least $27.6 \mathrm{~cm}$ apart, i.e. about 5 times the longest linear dimension of the device. On MICAz, they would still need to be $5 \mathrm{~cm}$ apart. A more reasonable multi-antenna 


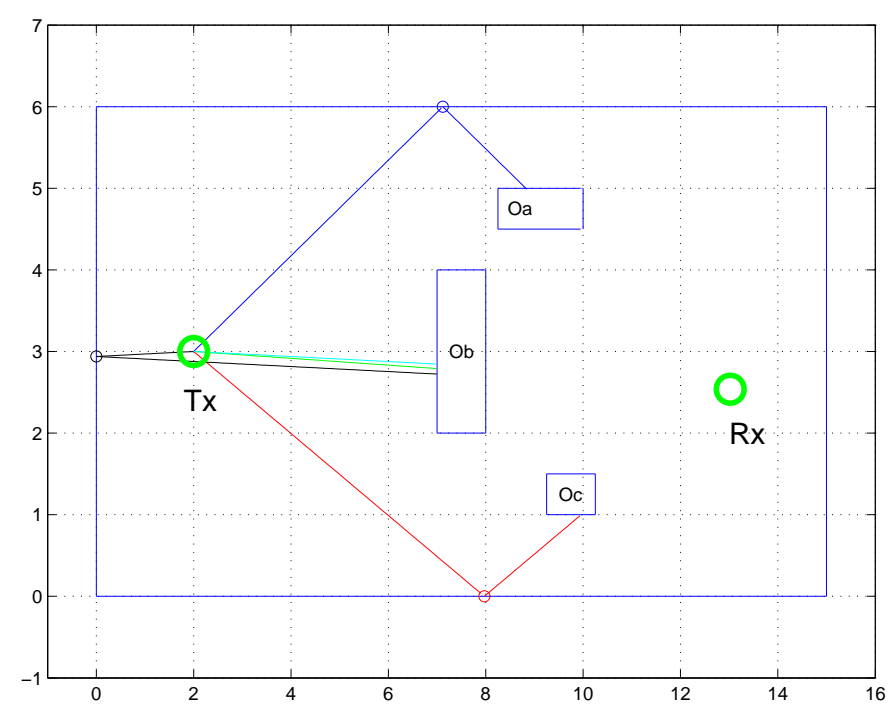

Fig. 6. Obstacles $O a, O b$, and $O c$ block off all multipath components.

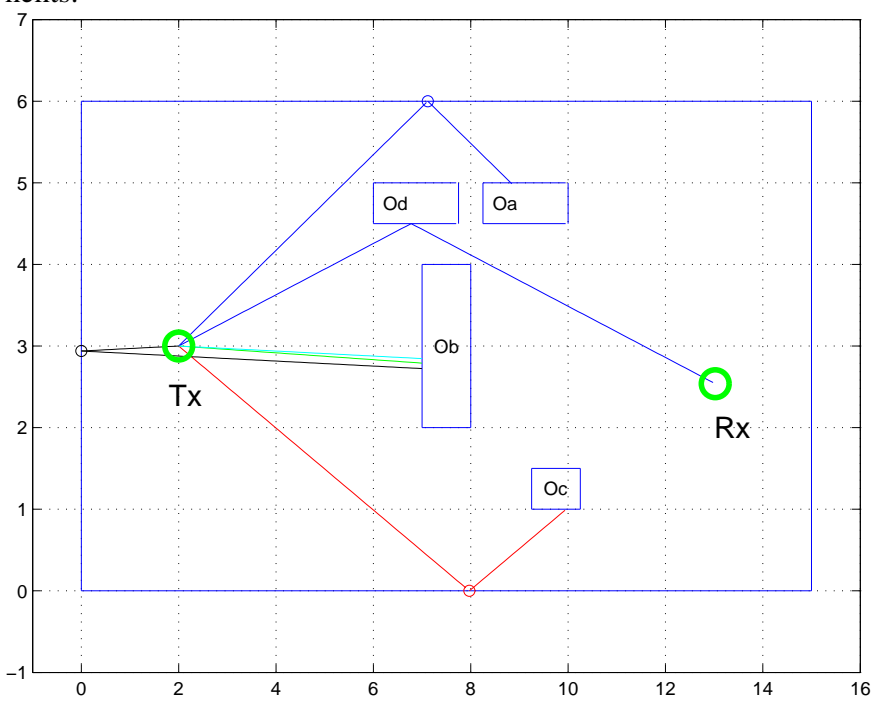

Fig. 7. Obstacle $O d$ produces a favorable fading patter which offsets the negative action of the other three obstacles.

solution is connecting multiple base stations together in a Master/Slave(s) architecture where the Master base station is wired to the gateway and is in charge of fusing the data coming from the Slave base station(s). In this case, however, a solution with $M$ antennae requires the coordinated use of $M$ devices.

We now compare a limited mobility and a multiantenna approach on the basis of experimental results. Figure 8 shows the layout of the room used for our experiment and a detail of the transmitter in the MISO (multiple transmit antennae and one receive antenna) and the limited mobility scenario. In the former, four MICAz motes are arranged in a circle of radius $\sqrt{2} \lambda / 4$

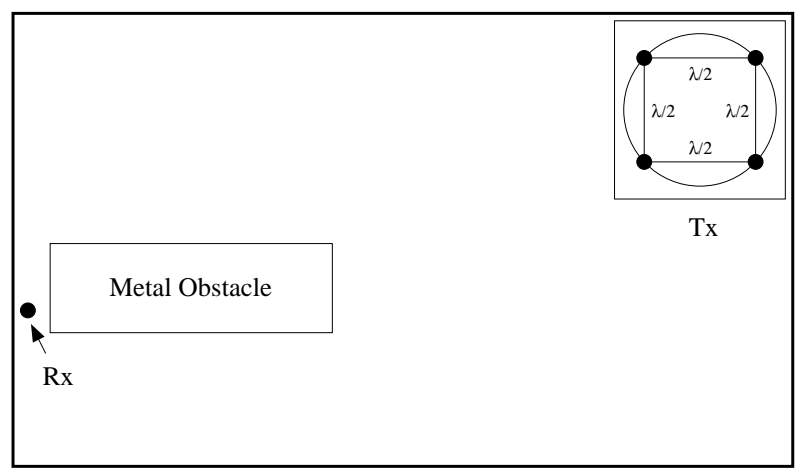

(a) MISO experiment.

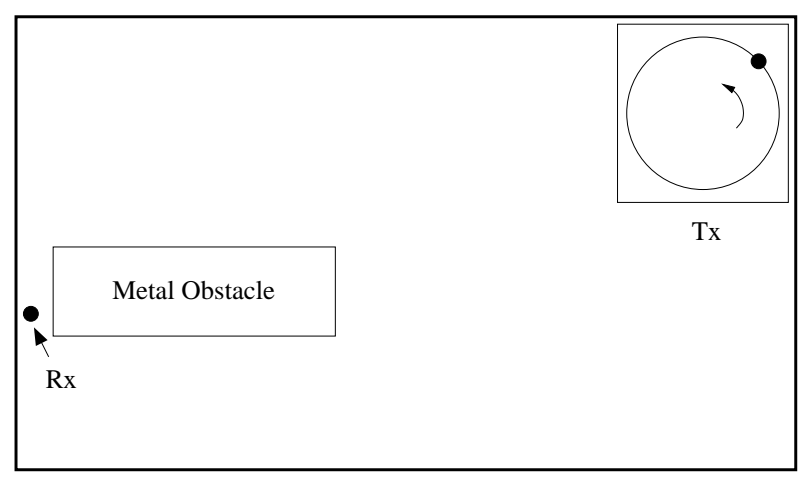

(b) Limited mobility experiment.

Fig. 8. Layout for the comparison of a multi-antenna and a limited mobility approach.

on top of a motorized turntable, so that nodes are $\lambda / 2$ apart. The motorized turntable is not moving in this part of the experiment. We implement a simple MISO system by scheduling each mote to send 1000 packets according to a round robin policy with a transmit power of $-10 \mathrm{dBm}$ to a receiver located about $8 \mathrm{~m}$ away. A large metal obstacle interrupts the LOS path; the typical received signal strength for this setup in this particular environment is between -85 and $-90 \mathrm{dBm}$, which is exactly the range of typical values for the reception threshold $\Theta$. We are right in the middle of the so-called transitional region [17], the range of received power in which links are highly unreliable.

When the four motes are placed on the vertices of the square in Figure 8(a), only one of the four links between the receiver and the multi-mote transmitter has a non-zero reception probability (it amounts to 17.8\%). On the other hand, limited mobility allows a continuous sampling of the path loss function, so that a particularly 


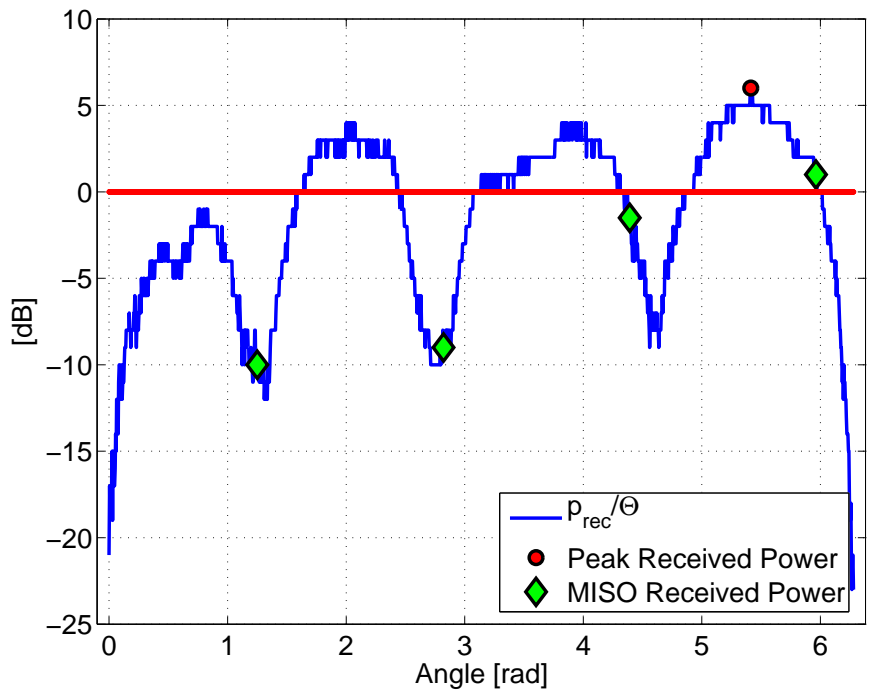

Fig. 9. With MISO systems, the reception probability depends on the position of the nodes. A mobile base station, on the other hand, can be opportunistically stopped at the position where the received power reaches its maximum. A multi-antenna system undersamples the path loss function, and a particularly unfavorable scenario like the one indicated in this figure (where only one antenna is barely above the reception threshold $\Theta$ ) is likely to occur.

favorable position can be located where the reception probability is as high as $97 \%$. We obtain this information by placing one mote on the circumference of the circle circumscribed to the square we considered (as shown in Figure 8(b)) and by letting the motorized turntable move slowly at the speed of about 3rpm (the radius of the turntable is $61 \mathrm{~cm})$. The turntable can be stopped once the received power is such that the desired reception probability is achieved, and the procedure can of course be made automatic by implementing a smart limited mobility system, i.e. a turntable that stops rotating once it finds the position that maximizes the reception probability. Figure 9 shows what happens in terms of signal strength. If the antennae of the MISO system are placed in the indicated spots, only one in four links exhibits non-zero packet reception. A different orientation of the same antenna array could yield better results, which indicates that the performance of a multi-antenna system is strongly dependent on the positioning of the antennae. The spatial undersampling operated by the multi-antenna approach can be clearly illustrated in terms of path loss functions. Over an area of the order of $\lambda^{2}$, the multiantenna approach seeks

$$
\max _{k=0 . . M-1} L_{p}\left(\mathbf{y}_{k}, \mathbf{j}\right),
$$

while the limited circular mobility approach looks for

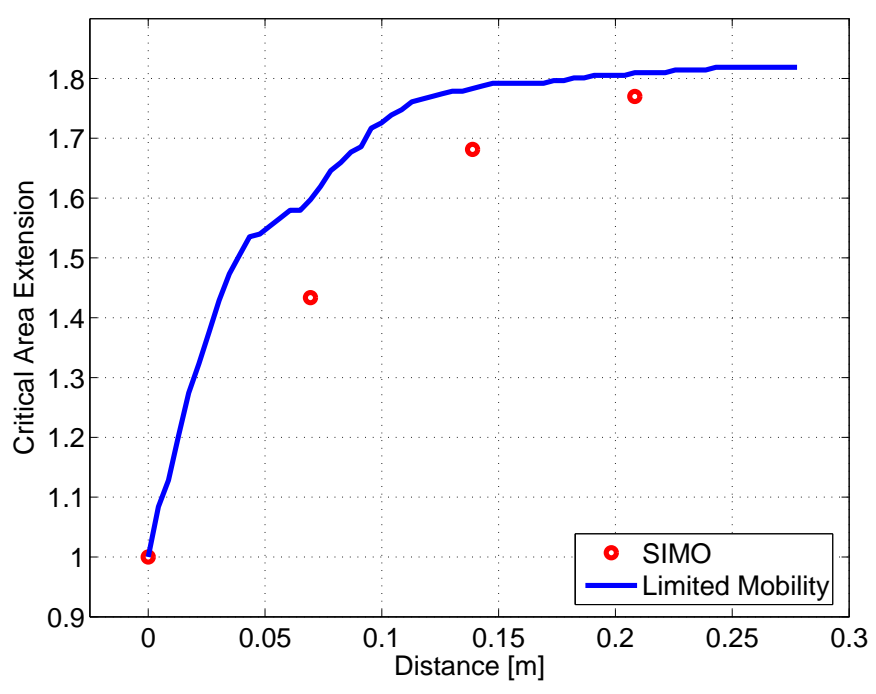

Fig. 10. Critical area extension in the multi-antenna and the limited mobility scenario. The horizontal axis represents the distance traveled by the mobile base station as it moves along a circumference of $\lambda \pi \sqrt{2} / 2$. We assume $\lambda=0.125 \mathrm{~m}$, so the circumference is $0.28 \mathrm{~m}$. The SIMO curve shows the critical area extension obtained with a system with one transmit antenna and four receive antennae placed $\lambda / 2$ apart as shown in Figure 8.

$$
\max _{0<t \leq T} L_{p}(\mathbf{y}(t), \mathbf{j}),
$$

where $T$ is the time it takes the node to complete one revolution along the circumference of a circle of radius $\sqrt{2} \lambda / 4$, and there exists a $t$ at which $L_{p}\left(\mathbf{y}_{k}, \mathbf{j}\right)=$ $L_{p}(\mathbf{y}(t+k T / 4), \mathbf{j})$ for $k=0 . . M-1$. The multi-antenna approach gives the maximum of $M$ samples of the path loss function, whereas the limited mobility approach returns the maximum of the function itself over the area that we consider.

We now use our geometric model to compare a SIMO approach (one transmit antenna, multiple receive antennae) to the limited mobility approach in the context of a network. We assume the absence of a LOS path $\left(s_{0}=0\right)$, consider an average attenuation coefficient $\bar{s}=$ 0.3 for paths 1 through 4 , fix a $20 \mathrm{~m} \times 20 \mathrm{~m}$ rectangular geometry, and set $\Theta$ at $-85 \mathrm{dBm}$. A popular model for node distribution is the homogenous Poisson point process (PPP) of intensity $\mu$ [5]. We use the critical area extension as a term of comparison, as it is proportional to the gain in network lifetime: if the size of the critical area grows larger, the traffic load directed to the base station can be distributed over more nodes. We simulate the SIMO case by letting the base station (located at the center of the deployment) move in angular steps of $\pi / 2$ along a circle of radius $\sqrt{2} \lambda / 4$, so that consecutive 
positions it occupies are spaced by $\lambda / 2$. We simulate the limited mobility scenario by having the base station move in a total of 64 angular steps of $\pi / 32$. Figure 10 shows the critical area extension in both scenarios, confirming that limited mobility is indeed a competitive strategy.

\section{CRITICAL AREA EXTENSION MODEL}

In this section, we study how the exploitation of spatial diversity relates to critical area extension in a wireless sensor network. We focus on a network with a manyto-one traffic pattern, where one base station seeks to collect data sent by a number of sensing nodes.

We will develop an analytical result to quantify the spatial diversity benefit in terms of critical area extension. Let $\mathcal{N}$ be the set of all nodes in the network, and let $\mathcal{N}_{i}$ be the subset of nodes that can be reached by the base station from the position of polar coordinates $\left(r_{i}, \theta_{i}\right)$. We define the dynamic critical area extension after $K$ steps as the ratio of the cardinality of the union of the subsets of $\mathcal{N}$ that can be reached from $K$ different positions of the base station and the cardinality $\mathcal{N}_{0}$, i.e., the number of nodes that can be reached in one-hop from the starting position:

$$
C_{K}=\frac{\# \cup_{i=0}^{K} \mathcal{N}_{i}}{\# \mathcal{N}_{0}} .
$$

The cardinalities of the sets $\mathcal{N}_{i}$ are random variables whose distribution depends on the network layout given the geometry of the environment. After setting a specific geometry, we can abstract from the position of the nodes by considering the expected critical area extension in $K$ steps $\mathbb{E}\left[C_{K}\right]$. With the help of the Rayleigh fading link model [4] and the Poisson random network abstraction, we can provide an analytical expression for $\mathbb{E}\left[C_{K}\right]$ given a circular geometry.

We assume the network nodes to be distributed according to a homogenous Poisson point process of unitary intensity. Given a circular area of radius $R$, the number of nodes is a Poisson-distributed random variable of mean $\pi R^{2}$; given that number, the nodes are uniformly distributed, and the distance $Z$ between the base station and the nodes has a probability density function of the form

$$
f_{Z}(z)=\frac{2 z}{R^{2}}, \quad z \in[0, R] .
$$

In an annulus formed by two concentric circles of radii $z$ and $z+d z$ centered at the origin, we expect to find a fraction of the total number of nodes given by $f_{Z}(z) d z$; each of them responds if the power it receives is higher than a certain threshold $\Theta$.

Assuming a flat Rayleigh fading channel, we have seen that the received power follows the exponential distribution given by (1); therefore, the reception probability given an inter-node distance $z$ is given by

$$
p_{\text {rec }}(z)=\exp \left(-\frac{\Theta z^{\alpha}}{P_{0}}\right) .
$$

Note that the Rayleigh fading assumption implies that no multipath component stands out at the base station, which is not the case in the presence of a dominating LOS component, which would call for a Ricean fading model [16]. The Rayleigh model characterizes a worstcase scenario (by encompassing the case of the absence of a LOS), and in addition to that is much more tractable than the Ricean model; these two reasons make it the most natural choice for the development of this framework.

Independently of the trajectory followed by the base station and further assuming that the channels seen by the base station at each step are independent, we may approximate the probability of correct reception at a distance $z$ from the origin after $K$ steps of the mobile sink as

$$
p_{\text {rec }}(z, K)=1-\left(1-\exp \left(-\frac{\Theta z^{\alpha}}{P_{0}}\right)\right)^{K+1}
$$

Since our focus is on limited mobility, we are also assuming the overall scope of the base station motion to be much smaller than the dimensions of the deployment $(\lambda K / 2 \ll R)$. Since $f_{Z}(z)$ gives the fraction of nodes located at distance $z$ from the mobile sink, we may estimate the expected critical area extension achieved in $K$ steps as

$$
\begin{aligned}
\mathbb{E}\left[C_{K}\right] & =1-\int_{0}^{R}\left(1-p_{\mathrm{rec}}(z, K)\right) f_{Z}(z) d z= \\
1 & -\int_{0}^{R} \frac{2 z}{R^{2}}\left(1-\exp \left(-\frac{\Theta z^{\alpha}}{P_{0}}\right)\right)^{K+1} d z \\
& =\left(\frac{P_{0}}{R^{\alpha} \Theta}\right)^{\frac{2}{\alpha}} \frac{2}{\alpha} \sum_{k=1}^{K+1} \frac{(-1)^{k} k^{\frac{\alpha-2}{\alpha}}(2+K-k)_{k}}{k !} \\
& \times\left(\Gamma_{\mathrm{ic}}\left(\frac{2}{\alpha}, \frac{k R^{\alpha} \Theta}{P}\right)-\Gamma_{\mathrm{c}}\left(\frac{2}{\alpha}\right)\right)
\end{aligned}
$$

where $(2+K-k)_{k}$ is a Pochhammer symbol ${ }^{1}$, and

\footnotetext{
${ }^{1}$ The Pochhammer symbol $(x)_{k}$ is shorthand for $\prod_{j=0}^{k-1}(x+j)$,
} and can also be written as $\frac{\Gamma_{\mathrm{c}}(x+k)}{\Gamma_{\mathrm{c}}(x)}$. 


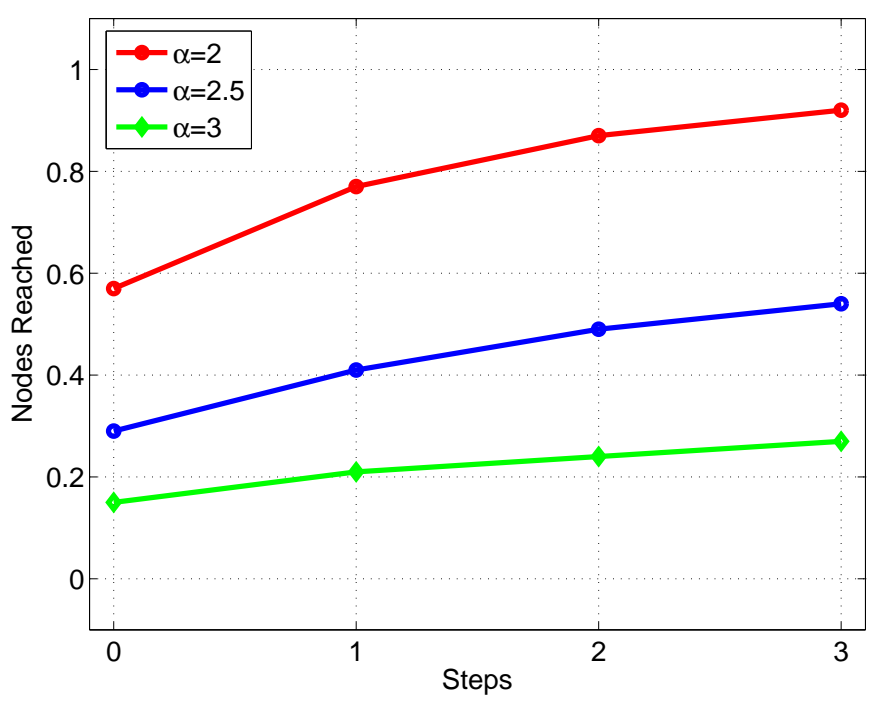

Fig. 11. Spatial diversity helps the base station reach more nodes. In this example, we study the motion of a node along a circle of radius $\sqrt{2} \lambda / 4$ in angular steps of $\pi / 2$, which models both a multiantenna and a limited mobility system. Here and in Figure 12 we set $R=11.3 \mathrm{~m}$.

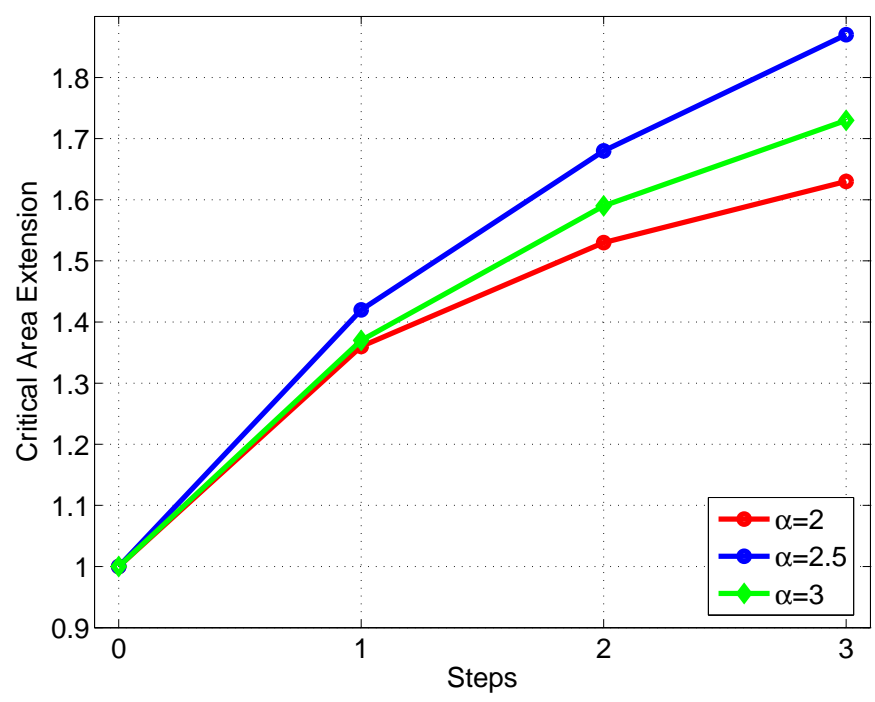

Fig. 12. The benefit in terms of critical area extension corresponding to the scenario in Figure $11(R=11.3 \mathrm{~m})$.

$\Gamma_{\mathrm{c}}$ and $\Gamma_{\text {ic }}$ are respectively the complete and incomplete gamma function $\left(\Gamma_{\mathrm{c}}(x)=\Gamma_{\mathrm{ic}}(x, 0)\right)$.

Note that this analysis also applies to a multi-antenna system with an array of $K+1$ receive antennae, which in this framework is indistinguishable from a base station moving in $K$ steps of $\lambda / 2$ (due to the assumption of independent channels). So $C_{K}$ may be interpreted as the critical area extension achieved with either $K$ steps or $K+1$ antennae.

Figure 11 shows the dynamic increase in the per-

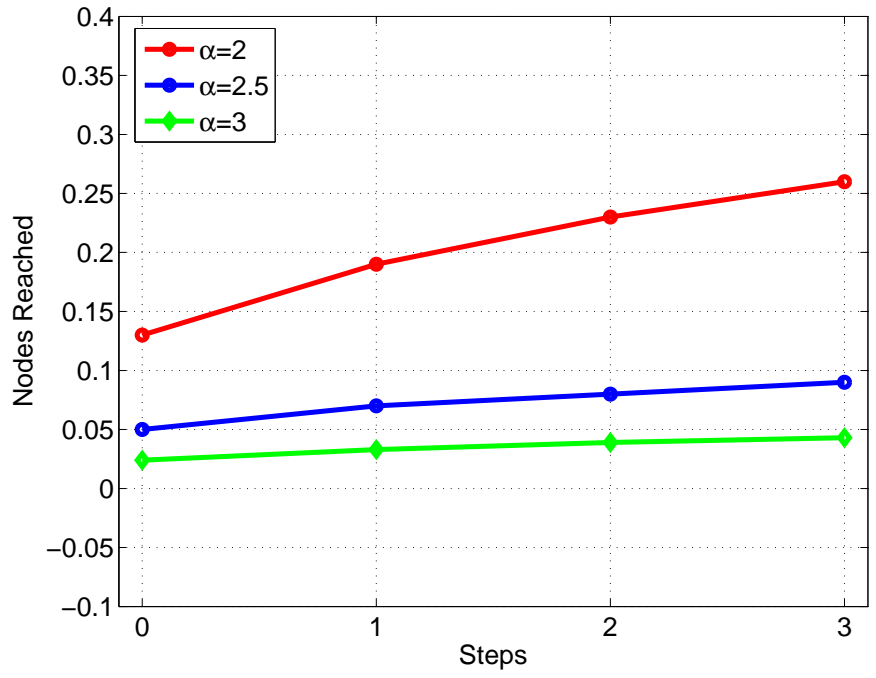

Fig. 13. Even with larger (here $R=28.2 \mathrm{~m}$ ) outdoor deployments and in the presence of harsh propagation conditions, spatial diversity still allows a small increase in the number of nodes which can be reached by the base station, which corresponds to the considerable critical area extension shown in Figure 14.

centage of nodes within the reach of the base station in various propagation conditions. Figure 12 shows that the spatial benefit can considerably extend the critical area, and therefore the lifetime of the network. Figures 13 and 14 show that such approaches are still valid in large outdoor scenarios. Even if the number of nodes that can dynamically reached by the base station corresponds to a small percentage of the total number of nodes in the network, the critical area extension is nonetheless considerable.

The extension of the critical area carries makes it possible to route over fewer (possibly longer) hops, which carries several advantages [18], such as the reduction of energy consumption at the sensing nodes, the achievement of a better energy balancing, the more aggressive exploitation of sleep modi, and the lack of route maintenance overhead. If nodes only occasionally need to act as relays, they can sleep longer and only consume energy to make their own data available.

\section{CLOSING REMARKS}

Despite fading is normally seen as negative, it can indeed be exploited constructively and opportunistically to communicate over channels that would otherwise be unusable. A definite element of novelty in the paper is the induction of fading through mobility as an alternative to its induction by means of multi-antenna systems introduced in [1]. Another interesting idea is the focus on limited mobility: whereas most mobility approaches 


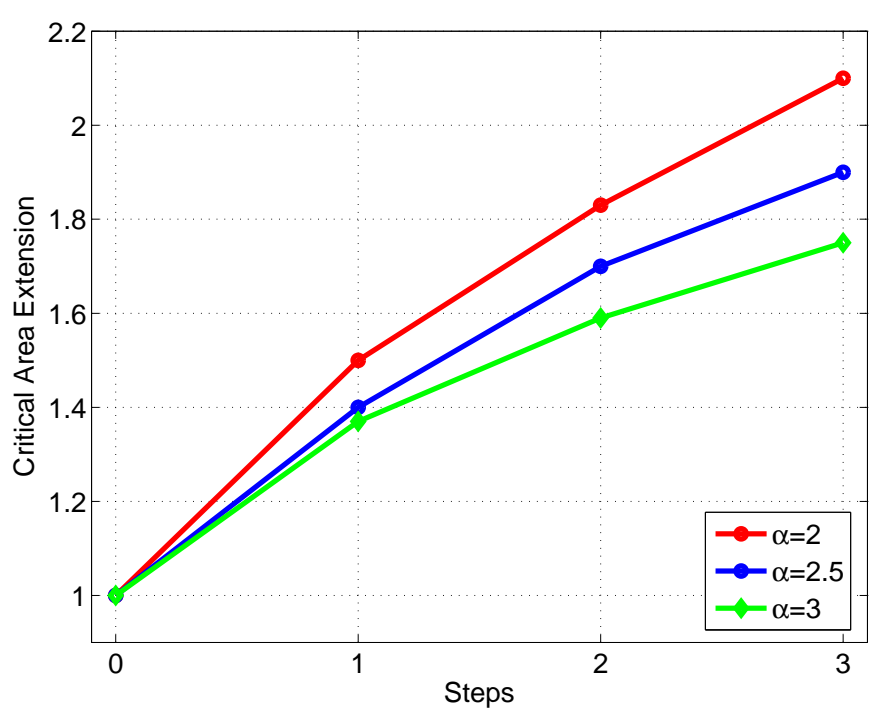

Fig. 14. The critical area extension due to limited mobility in large outdoor deployments $(R=28.2 \mathrm{~m})$ is quite considerable. Even if the propagation conditions are particularly harsh, a strong lifetime benefit can still be derived by the extension of the critical area.

tend to reduce the large scale path loss by letting a mobile agent get as close as possible to as many nodes as possible, our aim is to extend the number of singlehop neighbors of the base station by moving within an area of the order of $\lambda^{2}$.

After illustrating induced fading through measurements and numerical examples (using a simple geometric model), we have performed a comparison of multiantenna and limited mobility approaches with the help of experimental evidence. Limited mobility, though not necessarily easier or cheaper to implement than a multiantenna system, can provide greater benefits in terms of lifetime extension. Spacing multiple antennae at intervals of $\lambda / 2$ allows the exploitation of the spatial correlation properties of multipath fading, but limited mobility permits us to sample the path loss function at a higher rate and to abstract from the location of bad fading spots, with the guarantee of as good a performance as the wireless medium allows; on the other hand, the success of the multi-antenna approach is inherently reliant upon the specific physical location of the antennae.

Finally, we have developed analytical tools to relate spatial diversity to network lifetime by quantifying the extension of the critical area in a wireless sensor network with a statistical model that encompasses both the multiantenna and the limited mobility approach, showing that spatial diversity is a key direction for efforts aimed at network lifetime extension.

The work presented in this paper offers several pos- sibilities for future developments. In our experiments on limited mobility, we avail ourselves of a motorized turntable. One possibility is implementing the smart limited mobility system mentioned in this paper, in which a mobile receiver can choose the best fading spots on the basis of signal strength measurements and can stop moving accordingly. This would allow further investigations on the advantages of limited mobility over multi-antenna systems in the achievement of spatial diversity.

\section{ACKNOWLEDGEMENTS}

The support of the NSF (ECS03-29766 and CAREER CNS 04-47869) is gratefully acknowledged.

\section{REFERENCES}

[1] P. Viswanath, D. Tse, and R. Laroia. Opportunistic Beamforming Using Dumb Antennas. IEEE Transactions on Information Theory, 48(6):1277-1294, June 2002.

[2] M. Haenggi. Energy-Balancing Strategies for Wireless Sensor Networks. In IEEE International Symposium on Circuits and Systems (ISCAS'03), Bangkok, Thailand, May 2003.

[3] A. Goldsmith. Wireless Communications. Cambridge University Press, New York, NY, USA, 2005.

[4] M. Haenggi. On Routing in Random Rayleigh Fading Networks. IEEE Transactions on Wireless Communications, 4:1553-1562, 2005.

[5] M. Haenggi. A Geometry-Inclusive Fading Model for Random Wireless Networks. In 2006 IEEE International Symposium on Information Theory (ISIT'O6), Seattle, WA, USA, July 2006.

[6] K. Sohrabi, J. Gao, V. Ailawadhi, and G. Pottie. Protocols for self-organization of a wireless sensor network. IEEE Personal Communications Magazine, 7(5):16-27, October 2000.

[7] W. Zhao, M. Ammar, and E. Zegura. A message ferrying approach for data delivery in sparse mobile ad hoc networks. In The Fifth ACM International Symposium on Mobile Ad Hoc Networking and Computing (MobiHoc'04), Tokyo, Japan, May 2004.

[8] P. Venkitasubramaniam, S. Adireddy, and L. Tong. Sensor Networks with Mobile Access: Optimal Random Access and Coding. IEEE Journal on Selected Areas in Communications, 22(6):1058-1068, August 2004.

[9] L. Tong, Q. Zhao, and S. Adireddy. Sensor Networks with Mobile Agents. In Proceedings of the IEEE Military Communications Conference, Boston, MA, USA, 2003.

[10] J. Burrell, T. Brooke, and R. Beckwith. Vineyard Computing: Sensor Networks in Agricultural Production. IEEE Pervasive Computing, 3(1):38-45, 2004.

[11] R. C. Shah, S. Roy, S. Jain, and W. Brunette. Data MULEs: Modeling and analysis of a three-tier architecture for sparse sensor networks. In Ad Hoc Networks Journal, volume 1, pages 215-233. Elsevier, Sep 2003.

[12] W. Wang, V. Srinivasan, and K.-C. Chua. Using Mobile Relays to Prolong the Lifetime of Wireless Sensor Networks. In Proceedings of the 11th Annual International Conference on Mobile Computing and Networking, pages 270-283, Cologne, Germany, 2005.

[13] N. Amitay. Modeling and computer simulation of wave propagation in linear line-of-sight microcells. IEEE Transactions on Vehicular Technology, 41:337-342, 1992. 
[14] T. K. Sarkar, Z. Ji, K. Kim, A. Medour, and M. SalazarPalma. A Survey of Various Propagation Models for Mobile Communication. IEEE Antennas and Propagation Magazine, 45(3):51-82, June 2003.

[15] P. Gupta and P. R. Kumar. The Capacity of Wireless Networks. IEEE Transactions on Information Theory, 46:388-404, March 2005.

[16] W. C. Jakes. Microwave Mobile Communications. IEEE Press, New York, NY, USA, 1974.

[17] M. Zuniga and B. Krishnamachari. Analyzing the Transitional Region in Low Power Wireless Links. In Proceedings of the First IEEE International Conference on Sensor and Ad hoc Communications and Networks (SECON'04), Santa Clara, CA, USA, October 2004.

[18] Martin Haenggi and Daniele Puccinelli. Routing in Ad Hoc Networks: A Case for Long Hops. IEEE Communications Magazine, pages 93-101, October 2005. 